\title{
Detection of Coronavirus Disease (COVID-19) based on Deep Features and Support Vector Machine
}

\author{
Prabira Kumar Sethy \\ Department of Electronics, \\ Sambalpur University, Odisha, India. \\ Corresponding author: prabirasethy@ suiniv.ac.in
}

Santi Kumari Behera

Department of Computer Science and Engineering, Veer Surendra Sai University of Technology, Odisha, India.

E-mail: santikbehera_cse@vssut.ac.in

Pradyumna Kumar Ratha

Department of Computer Science and Engineering, Sambalpur University Institute of Information Technology, Sambalpur University, Odisha, India.

E-mail: pkrath@suiit.ac.in

\section{Preesat Biswas}

Department of Electronics and Communication Engineering, Dr. C. V. Raman University, Chhattisgarh, India.

E-mail: preesat.eipl@gmail.com

(Received April 1, 2020; Accepted April 15, 2020)

\begin{abstract}
The detection of coronavirus (COVID-19) is now a critical task for the medical practitioner. The coronavirus spread so quickly between people and approaches 100,000 people worldwide. In this consequence, it is very much essential to identify the infected people so that prevention of spread can be taken. In this paper, the deep feature plus support vector machine (SVM) based methodology is suggested for detection of coronavirus infected patient using X-ray images. For classification, SVM is used instead of deep learning based classifier, as the later one need a large dataset for training and validation. The deep features from the fully connected layer of CNN model are extracted and fed to SVM for classification purpose. The SVM classifies the corona affected X-ray images from others. The methodology consists of three categories of Xray images, i.e., COVID-19, pneumonia and normal. The method is beneficial for the medical practitioner to classify among the COVID-19 patient, pneumonia patient and healthy people. SVM is evaluated for detection of COVID-19 using the deep features of different 13 number of CNN models. The SVM produced the best results using the deep feature of ResNet50. The classification model, i.e. ResNet50 plus SVM achieved accuracy, sensitivity, FPR and F1 score of 95.33\%,95.33\%,2.33\% and 95.34\% respectively for detection of COVID-19 (ignoring SARS, MERS and ARDS). Again, the highest accuracy achieved by ResNet50 plus SVM is $98.66 \%$. The result is based on the Xray images available in the repository of GitHub and Kaggle. As the data set is in hundreds, the classification based on SVM is more robust compared to the transfer learning approach. Also, a comparison analysis of other traditional classification method is carried out. The traditional methods are local binary patterns (LBP) plus SVM, histogram of oriented gradients (HOG) plus SVM and Gray Level Co-occurrence Matrix (GLCM) plus SVM. In traditional image classification method, LBP plus SVM achieved $93.4 \%$ of accuracy.
\end{abstract}

Keywords- Coronavirus, COVID-19, Diagnosis, Deep features, SVM. 
International Journal of Mathematical, Engineering and Management Sciences

Vol. 5, No. 4, 643-651, 2020

https://doi.org/10.33889/IJMEMS.2020.5.4.052

\section{Introduction}

Nowadays, "coronavirus" is prefixed with the word "novel," as it is a new strain of the virus. As indicated by the WHO, coronaviruses have a place with a wide family run from the usual cold to perilous illnesses (World Health Organization, 2020). The strain that began spreading in Wuhan, the capital of China's Hubei locale, is recognized from two distinctive coronaviruses, namely severe acute respiratory syndrome (SARS) and the Middle East respiratory syndrome (MERS). Indications of coronavirus defilement go in earnestness from respiratory complexity like pneumonia, kidney issue and development of fluid in the lungs.

On $11^{\text {th }}$ February 2020, the WHO Director-General gave an acronym "COVID-19" to these infections were found to be caused by a new coronavirus. In the last two decades, two coronavirus epidemics are observed, i.e. SARS-CoV and MERS-CoV. The first one started in china, spread to twenty-four countries and reported 8000 cases \& 800 of deaths. The second one started from Saudi Arabia, reported 2500 cases and 800 deaths. The detail of coronavirus is depicted in Table 1.

Table 1. Detail of coronavirus

\begin{tabular}{cccc}
\hline CoV & Year & Origin & Mortality rate \\
\hline SARS & 2002 & Guangdong province, China & $10 \%$ \\
MERS & 2013 & Saudi Arabia & $34 \%$ \\
COVID-19 & 2019 & Wuhan, China & $3.4 \%$ \\
\hline
\end{tabular}

Among the causing pathogens for respiratory diseases, $\mathrm{CoV}$ is become the dangerous one because of its serial interval (5 to 7.5) and reproductive rate (2 to 3) (Nishiura et al., 2020). The CoV belongs to single-stranded RNA viruses (+ssRNA) family mostly observed in animals (Perlman and Netland, 2009; Chan et al., 2013). The analysis carried out till date, the viruses have no species barrier and can cause severe diseases like MERS and SARS. The coronavirus infection can provoke SARS that is severe enough to be called Acute respiratory distress syndrome (ARDS). In general, estimates suggest that $2 \%$ of the population are healthy carriers of a $\mathrm{CoV}$ and that these viruses are responsible for about 5\% to $10 \%$ of acute respiratory infections (Chen et al., 2020a). COVID-19 spreads more quickly than SARS and have symptoms like other coronaviruses. Figure 1 shows the distribution of COVID-19 cases and deaths worldwide, as of $13^{\text {th }}$ April 2020 (European Centre for Disease Prevention and Control, 2020).

The test of COVID-19 is currently a difficult task because of the unavailability of the diagnosis system everywhere, which is causing panic. On account of the restricted accessibility of COVID19 testing units, we have to depend on different finding measures. Since COVID-19 attacks the epithelial cells that line our respiratory tract, we can utilize Xray to examine the strength of lungs. The medical practitioner often uses Xray images to diagnose any kind of lungs diseases. Furthermore, nearly in all emergency clinics have X-beam imaging machines, it could be conceivable to utilize Xray to test for COVID-19 without the devoted test kits. Therefore, developing an automated analysis system is necessary to save medical professionals valuable time. 
International Journal of Mathematical, Engineering and Management Sciences

Vol. 5, No. 4, 643-651, 2020

https://doi.org/10.33889/IJMEMS.2020.5.4.052

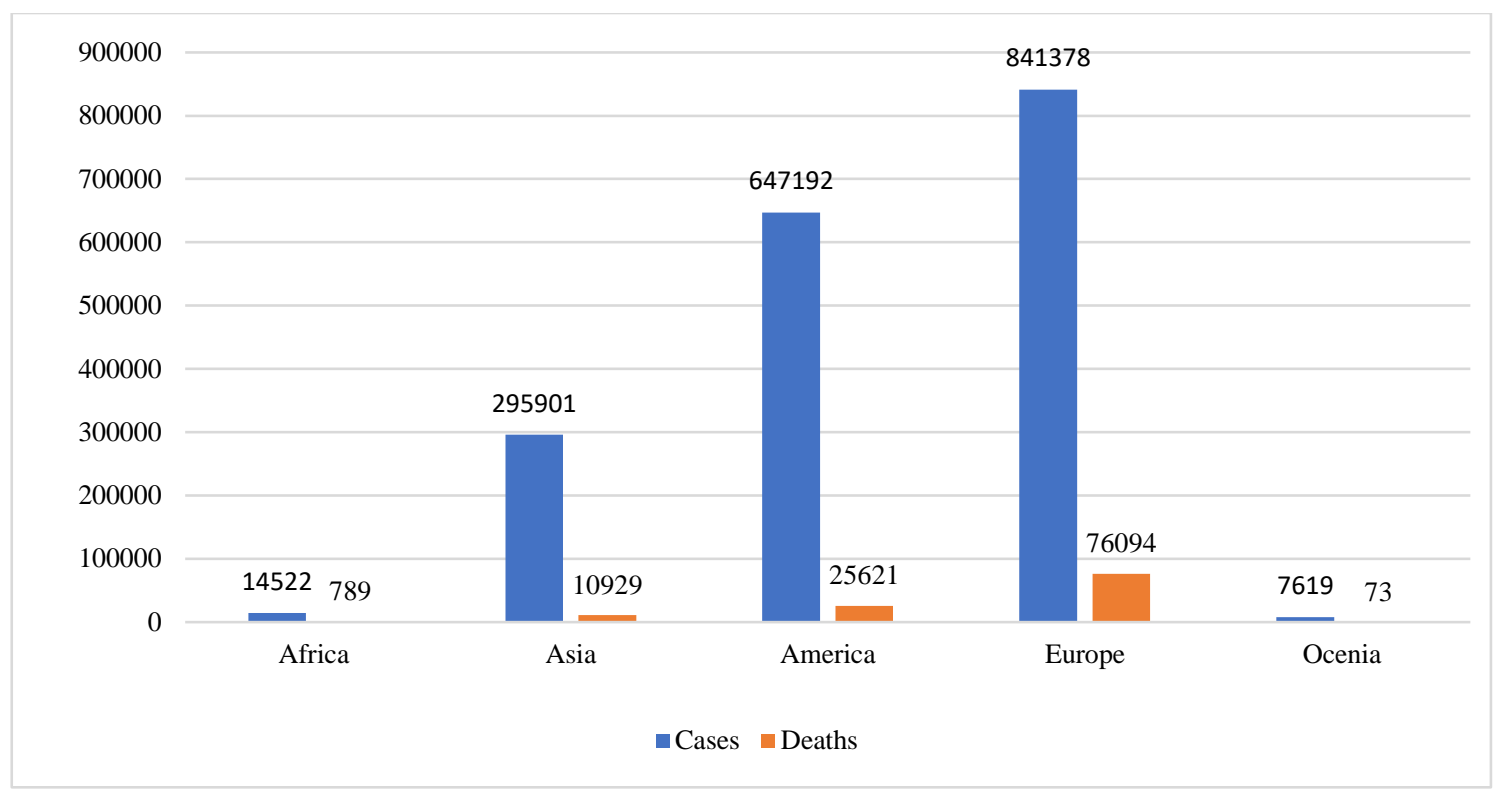

Figure 1. COVID-19 cases and deaths worldwide, as of $13^{\text {th }}$ April 2020

In the current situation of the rapid spread of COVID-19 many kinds of research have been going on based on clinical features (Chen et al., 2020b; Wang et al., 2020; Yang et al., 2020). Deep Learning is a combination of machine learning methods that mainly focused on the automatic feature extraction and classification from images. The object detection and classification are the two main tasks where deep learning is applied. The advancement of machine learning has a great benefit for clinical decision making and development computer-aided systems (Greenspan et al., 2016; Burlina et al., 2017; Wahab et al., 2017; Xia et al., 2018). As the data set available is in hundreds, which is very small for transfer learning approach and it's leading a question mark about the robustness of the classification model. So, instead of using a pre-trained network as a classifier in transfer learning approach to detect the COVID-19, we choose SVM as the classifier. The SVM classifies Xray images of COVID-19 patient, pneumonia patient and healthy people with the use of deep features extracted from fully connected layer of the pre-trained network.

In this paper, a classification model is developed, which classify the Xray images of COVID-19, pneumonia patient and healthy people. The main objective of this research is to screen out the healthy people and pneumonia patient. The healthy people are in doubt because they came from abroad or, previously living in a COVID-19 affected area. Again, mostly pneumonia symptoms and COVID-19 are some extents similar, and both are lungs related diseases. So, it also needs to screen out the pneumonia patient from COVOD-19. Hence, in this study, we prepared a dataset contains Xray images of COVID-19 patient, pneumonia patient and healthy people. The dataset consists of 127 confirmed COVID-19 images, 127 confirmed pneumonia images and 127 healthy images. i.e. in total 381. Again, the pneumonia images include 63 bacterial pneumonia and 64 viral pneumonia. The COVID-19 X-ray images are collected from the GitHub repository shared by Dr. Joseph Cohen, a postdoctoral fellow at the University of Montreal (Cohen et al., 2020). The GitHub repository contains Xray and CT images of SARS, MERS, and Pneumonia with the frontal and lateral view. Again, we exclude the MERS, SARS, and ARDS. We only choose 48 COVID-19 frontal view Xray images. The frontal view includes posteroanterior (PA) and anteroposterior (AP) 
International Journal of Mathematical, Engineering and Management Sciences

Vol. 5, No. 4, 643-651, 2020

https://doi.org/10.33889/IJMEMS.2020.5.4.052

views. Mostly these views are used to examine the lung diseases. In addition to GitHub, we collected 79 Xray images from Kaggle repository (Kaggle, 2020). Again, another two sets of Xray images (pneumonia and healthy) are collected from (Kermany et al., 2018) to train the classification model to distinguish the COVID-19 from pneumonia patient and healthy people. The Xray images of COVID-19, Bacterial Pneumonia, Viral Pneumonia and Normal are shown in Figure 2. The detail about the dataset is in Table 2 .

We use this dataset for deep feature extraction using pre-trained networks such as AlexNet, VGG16, VGG19, GoogleNet, ResNet18, ResNet50, ResNet101, InceptionV3, InceptionResNetV2, DenseNet201, XceptionNet, MobileNetV2 and ShuffleNet. The deep features obtained from these networks are fed to the SVM for classification. Again, the traditional image classification methods are applied for the detection of COVID-19.

Table 2. Detail of dataset

\begin{tabular}{llclc}
\hline & \multicolumn{1}{c}{ Samples } & Number & Repository & Total \\
\hline COVID-19 & $\begin{array}{l}\text { COVID-19 } \\
\text { without MERS, SARS, ARDS } \\
\text { (Frontal View Xray images) }\end{array}$ & 48 & GitHub (Dr. Joseph Cohen) & 127 \\
& COVID-19 & 79 & Kaggle & \\
& Bacterial Pneumonia & 63 & Kermany et al. 2018 & 127 \\
\hline Pneumonia & Viral Pneumonia & 64 & & 127 \\
\hline Normal & & 127 & & 127 \\
\hline
\end{tabular}

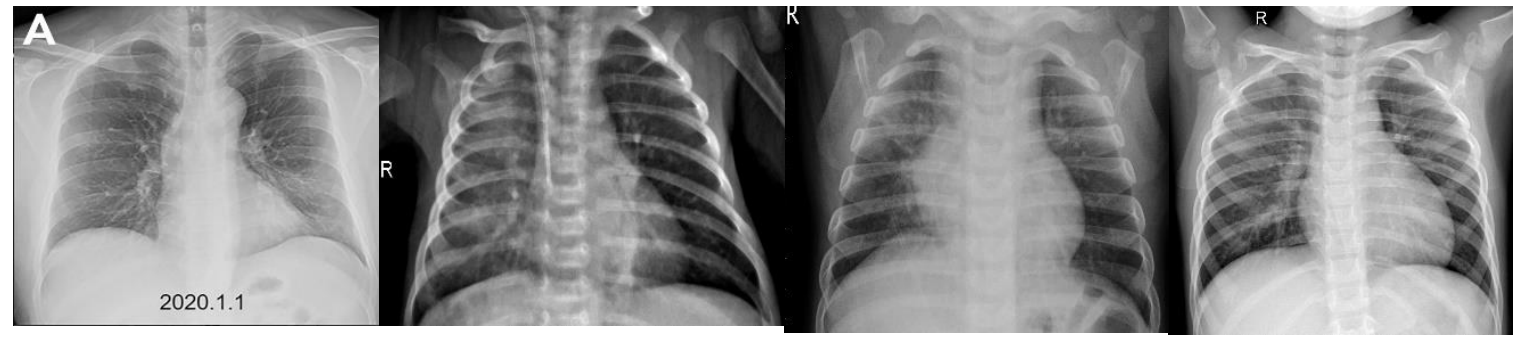

(a)

(b)

(c)

(d)

Figure 2. Xray images (a) COVID-19 (b) Bacterial pneumonia (c) Viral pneumonia (d) Normal

The main contribution of this article is as follows.

- We prepared a dataset of 381 Xray images, i.e. chest Xray frontal view images of 127 COVID19, 127 pneumonia and 127 healthy people.

- The deep features of most used 13 number of deep CNN models (AlexNet, VGG16, VGG19, GoogleNet, ResNet18, ResNet50, ResNet101, InceptionV3, InceptionResNetV2, DenseNet201, XceptionNet, MobileNetV2 and ShuffleNet) are extracted and used by SVM classifier for detection of COVID-19.

- The proposed method is a three-class problem to classify the Xray images of healthy people, pneumonia patient and COVID-19 patient. 
International Journal of Mathematical, Engineering and Management Sciences

Vol. 5, No. 4, 643-651, 2020

https://doi.org/10.33889/IJMEMS.2020.5.4.052

- Finally, a comparative analysis of deep feature plus SVM and traditional image classification method (LBP+SVM, HOG+SVM and GLCM+SVM) is carried out.

The remaining paper is organised as follow. Section 2 discussed the methodology. The experimental results are detailed in section 3 . At last, section 4 concludes with future scope.

\section{Methodology}

Deep feature extraction is based on the extraction of features acquired from a pre-trained CNN (Lopes and Valiati, 2017). The deep features are extracted from fully connected layer and feed to the classifier for training purpose. The deep features obtained from each CNN networks are used by SVM classifier. After that, the classification is performed, and the performance of all classification models are measured. The COVID-19 disease detection model based on deep features by SVM classifier is shown in Figure 3.

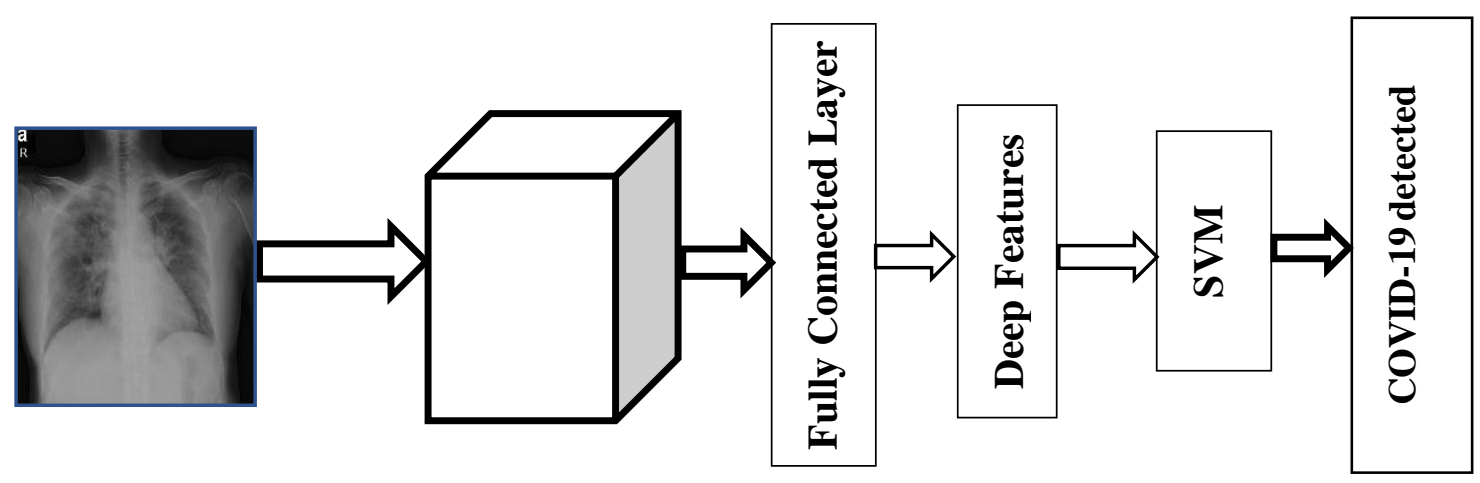

Figure 3. Detection COVID-19 by SVM based on deep feature using X-ray images

The deep features of CNN models are extracted from a particular layer and feature vector is obtained. The features are fed to the SVM classifier for classification of COVID-19, pneumonia patient and healthy people. The CNN is multilayer structure network, and each layer produces a response. The layers extract the essential image feature and pass to the next layer. The feature layer and feature vector used by CNN models are detailed in Table 3. The activation is in GPU with a minibatch size of 64 and GPU memory have space enough to fit image dataset. The activation output is in the form of the column to fit in linear SVM training. To train the SVM, the function 'fit class error-correcting output codes ('fitcecoc') is used. This function returns full trained multiclass error-correcting output of the model. The function 'fitcecoc' uses $\mathrm{K}(\mathrm{K}-1) / 2$, binary SVM model, using One-Vs-All coding design. Here, $\mathrm{K}$ is a unique class label. Because of error-correcting output codes and one-Vs-all coding design of SVM, the performance of classification models is enhanced.

In the convolution layer, formats of enrolled channels are utilised. Each one channel is limited spatially (traverse along with height and weight) but enlarges with the complete deepness of input volume. The images that have, Height $\mathrm{H}$, Depth D and Width $\mathrm{W}$ shading channels (i.e., $\mathrm{H} \times \mathrm{D}, \mathrm{W}$ ), the enrolled channels isolate an image width as $W 1=\frac{(W-F+2 p)}{S+1}$, here $\mathrm{F}$ speaks to the spatially expands neuron estimate; $p$ is the main part of zero padding, and $S$ is the size of way. Thus, the 
International Journal of Mathematical, Engineering and Management Sciences

Vol. 5, No. 4, 643-651, 2020

https://doi.org/10.33889/IJMEMS.2020.5.4.052

height is partitioned by $H 1=\frac{(H-F+2 p)}{S+1}$; depth D1 is the extent of the number of channels $\mathrm{K}$. For instance, an image having $28 \times 28 \times 3$ ( 3 is for the shading channels), if the open field (or channel) has a size of $5 \times 5 \times 3$ (altogether 75 neurons +1 bias), a $5 \times 5$ window with profundity three moves along the width and height and produces a 2-D activation map. The Pooling Layer works individually above all the deepness portion for the input and rescales it extensional applying the MAX operation. It obtained the size of the volume of HDW and separates the image into $W 1=$ $\frac{W-F}{S+1}$ as Width and $H 1=\frac{H-F}{S+1}$ as Stature and profundity D1 is same as the info D. After the calculation against each shading channels the MAX task is finished. In this way, the feature matrix is then diminished in POOLING layer.

Table 3. Details of feature layer and feature vector of CNN models

\begin{tabular}{llllll}
\hline $\begin{array}{l}\text { CNN } \\
\text { models }\end{array}$ & Feature Layer & Feature Vector & $\begin{array}{l}\text { CNN } \\
\text { models }\end{array}$ & $\begin{array}{l}\text { Feature } \\
\text { Layer }\end{array}$ \\
\hline AlexNet & fc6 & 4096 & Xception & predictions \\
Vector
\end{tabular}

\section{Results and Discussion}

In this study, we examined the performance of classification models for detection of COVID-19 based on eleven number of CNN models. The experimental studies were implemented using the MATLAB 2019a deep learning toolbox. All applications were run on a laptop, i.e. Acer Predator Helios 300 Core i5 8th Gen - (8 GB/1 TB HDD/128 GB SSD/Windows 10 Home/4 GB Graphics) and equipped with NVIDIA GeForce GTX 1050Ti. The measurement of performance of each classifier is measured in terms of Accuracy, Sensitivity, Specificity, False positive rate (FPR), F1 Score, MCC and Kappa. In addition, this experimentation used One-Vs-all approach and linear SVM as the SVM classifier parameter. The results reported in Table 4 and Table 5 are based on the average value of 20 independent executions. The training and testing ratio is 80:20 and adapted randomized selection for training and testing in each execution. The accuracy of different classification models with their mean, minimum and maximum achievable values in 20 independent execution are recorded in Table 4. Also, the other performance measures such as sensitivity, FPR and F1 score of different classification models are recorded in Table 5.

It is observed from Table 4, the accuracy of ResNet50 plus SVM is better among the 13 classification models. The mean, minimum and maximum value of accuracy is higher than the other classification models, i.e. $95.33 \%, 92.00 \%$ and $98.66 \%$ respectively. Again, it is observed from Table 5, the ResNet50 plus SVM results in higher sensitivity and F1 Score, i.e. 95.33\% and 95.34\% respectively. The most important parameter for measuring the performance of any classification model is the false positive rate (FPR) and need to be minimum. Here, the ResNet50 plus SVM have the lowest FPR, i.e. $2.33 \%$. 
International Journal of Mathematical, Engineering and Management Sciences

Vol. 5, No. 4, 643-651, 2020

https://doi.org/10.33889/IJMEMS.2020.5.4.052

Table 4. Accuracy (\%) of different classification models based on SVM using deep features of various CNN models

\begin{tabular}{lccc}
\hline \multicolumn{1}{c}{ Classification Models } & Mean & Minimum & Maximum \\
\hline AlexNet & 94.86 & 90.66 & 98.66 \\
DenseNet201 & 93.86 & 88.00 & 97.33 \\
GoogleNet & 91.73 & 84.00 & 98.66 \\
InceptionResnetV2 & 91.13 & 81.33 & 97.33 \\
InceptionV3 & 90.26 & 85.33 & 96.00 \\
MobileNetV2 & 94.46 & 90.66 & 98.66 \\
ResNet18 & 94.26 & 89.33 & 98.66 \\
XceptionNet & 93.00 & 88.00 & 97.33 \\
ResNet101 & 93.53 & 88.00 & 98.66 \\
ShuffleNet & 65.26 & 60.00 & 70.66 \\
VGG16 & 94.20 & 92.00 & 97.33 \\
VGG19 & 94.13 & 89.33 & 98.66 \\
ResNet50 & 95.33 & 92.00 & 98.66 \\
\hline
\end{tabular}

Table 5. Performance measures (\%) in terms of sensitivity, FPR and F1 score of different classification models based on SVM using deep features of various CNN models

\begin{tabular}{lccc}
\hline \multicolumn{1}{c}{ Classification Models } & Sensitivity & FPR & F1 Score \\
\hline AlexNet & 94.86 & 2.56 & 94.85 \\
DenseNet201 & 93.86 & 3.06 & 93.86 \\
GoogleNet & 91.73 & 4.13 & 91.74 \\
InceptionResnetV2 & 91.13 & 4.43 & 91.04 \\
InceptionV3 & 90.26 & 4.86 & 90.28 \\
MobileNetV2 & 94.46 & 2.76 & 94.46 \\
ResNet18 & 94.26 & 2.86 & 94.25 \\
XceptionNet & 93.00 & 3.50 & 93.00 \\
ResNet101 & 93.53 & 3.23 & 93.54 \\
ShuffleNet & 65.26 & 17.36 & 58.79 \\
VGG16 & 94.20 & 2.90 & 94.20 \\
VGG19 & 94.13 & 2.93 & 94.15 \\
ResNet50 & $\mathbf{9 5 . 3 3}$ & $\mathbf{2 . 3 3}$ & $\mathbf{9 5 . 3 4}$ \\
\hline
\end{tabular}

\subsection{Comparison with Other Machine Learning Approach}

In image processing and machine learning approach, mostly HOG plus SVM, GLCM plus SVM and LBP plus SVM are applied for image classification. The accuracy of those approaches is given in Table 6 .

Table 6. Accuracy (\%) score of traditional image classification methods

\begin{tabular}{cccc}
\hline Methods & GLCM+SVM & HOG+SVM & LBP+SVM \\
\hline Accuracy & 93.2 & 88.5 & 93.4 \\
\hline
\end{tabular}

\subsection{Discussion and Comparison of Simulation Results}

The proposed study used pre-trained CNN models to obtain the best performance for detection of COVID-19. We evaluated the performance results of deep feature extraction based on the AlexNet, VGG16, VGG19, GoogleNet, DenseNet201, InceptionResNetV2, InceptionV3, ResNet18, ResNet50, ReseNet101, XceptionNet, MobileNetV2 and ShuffleNet deep models. Also, traditional methods such as LBP plus SVM, HOG plus SVM, and GLCM plus SVM, which are widely used in object recognition, were applied, and their accuracy scores were evaluated. 
International Journal of Mathematical, Engineering and Management Sciences

Vol. 5, No. 4, 643-651, 2020

https://doi.org/10.33889/IJMEMS.2020.5.4.052

First, we extract deep features from a specific layer using the AlexNet, VGG16, VGG19, GoogleNet, DenseNet201, InceptionResNetV2, InceptionV3, ResNet18, ResNet50, ReseNet101, XceptionNet, MobileNetV2 and ShuffleNet pre-trained networks. The specific feature layer and its feature vector are detailed in Table 3. The obtained deep features were calculated for their performance using SVM classifier. According to the results, the ResNet50 plus SVM model achieved the highest classification accuracy, i.e. 95.33\%.

Later, traditional methods such as LBP plus SVM, HOG plus SVM and GLCM plus SVM were evaluated for detection of COVID-19. According to the results, among these three methods, the highest classification accuracy was $93.4 \%$ using the LBP plus SVM classification model.

\section{Conclusion}

The content of the manuscript about the coronavirus is based on the data available in WHO, European Centre for Disease Prevention and Control An agency of the European Union and other official websites worldwide. The chest X-ray images are used for simulation purposes are collected from GitHub and Kaggle. The adapted methodology for detection of coronavirus (COVOD-19) using X-ray images is based on deep feature and SVM. For this, we extracted the deep feature of 13 pre-trained CNN model and fed to SVM classifier individually. For better robustness of the classification model, each classification model is executed 20 times, and the average value is recorded. The classification model, i.e. ResNet50 plus SVM, is performing better compared to other 12 classification models. The proposed classification model for detection of COVID-19 is achieved $95.33 \%$ of accuracy. The accuracy of $95.33 \%$ is the average of 20 independent executions, and its highest achieved value of accuracy is $98.66 \%$. This research may be extended with a large dataset. The limitation of this methodology is that if the patient is in a critical situation and unable to attend for Xray scanning.

\section{Conflict of Interest}

The authors confirm that there is no conflict of interest to declare for this publication.

\section{Acknowledgments}

This research did not receive any specific grant from funding agencies in the public, commercial, or not-for-profit sectors. The authors sincerely appreciate the editor and reviewers for their time and valuable comments.

\section{References}

Chan, J.F.W., To, K.K.W., Tse, H., Jin, D.Y., \& Yuen, K.Y. (2013). Interspecies transmission and emergence of novel viruses: lessons from bats and birds. Trends in Microbiology, 21(10), 544-555.

Chen, N., Zhou, M., Dong, X., Qu, J., Gong, F., Han, Y., \& Yu, T. (2020b). Epidemiological and clinical characteristics of 99 cases of 2019 novel coronavirus pneumonia in Wuhan, China: a descriptive study. The Lancet, 395(10223), 507-513.

Chen, Y., Liu, Q., \& Guo, D. (2020a). Emerging coronaviruses: genome structure, replication, and pathogenesis. Journal of Medical Virology, 92(4), 418-423.

Cohen, J.P., Morrison, P., \& Dao, L. (2020). COVID-19 image data collection. arXiv preprint arXiv:2003.11597. Link: https://github.com/ieee8 023/covid-chestxray-dataset. 
International Journal of Mathematical, Engineering and Management Sciences

Vol. 5, No. 4, 643-651, 2020

https://doi.org/10.33889/IJMEMS.2020.5.4.052

European Centre for Disease Prevention and Control. An agency of the European Union. Link: https://www.ecdc.europa.eu/en/geographical-distribution-2019-ncov-cases.

Greenspan, H., Van Ginneken, B., \& Summers, R.M. (2016). Guest editorial deep learning in medical imaging: overview and future promise of an exciting new technique. IEEE Transactions on Medical Imaging, 35(5), 1153-1159.

Kaggle. Link: https://www.kaggle.com/andrewmvd/convid19-X-rays.

Kermany, D.S., Goldbaum, M., Cai, W., Valentim, C.C., Liang, H., Baxter, S.L., \& Dong, J. (2018). Identifying medical diagnoses and treatable diseases by image-based deep learning. Cell, 172(5), 11221131.

Lopes, U.K., \& Valiati, J.F. (2017). Pre-trained convolutional neural networks as feature extractors for tuberculosis detection. Computers in Biology and Medicine, 89, 135-143.

Nishiura, H., Linton, N.M., \& Akhmetzhanov, A.R. (2020). Serial interval of novel coronavirus (COVID19) infections. International Journal of Infectious Diseases, 93, 284-286.

Perlman, S., \& Netland, J. (2009). Coronaviruses post-SARS: update on replication and pathogenesis. Nature Reviews Microbiology, 7(6), 439-450.

Wahab, N., Khan, A., \& Lee, Y.S. (2017). Two-phase deep convolutional neural network for reducing class skewness in histopathological images based breast cancer detection. Computers in Biology and Medicine, 85, 86-97.

Wang, D., Hu, B., Hu, C., Zhu, F., Liu, X., Zhang, J., \& Zhao, Y. (2020). Clinical characteristics of 138 hospitalized patients with 2019 novel coronavirus-infected pneumonia in Wuhan, China. Jama, 323(11), 1061-1069.

World Health Organization. (2020, April 13). Link: https://www.who.int/emergencies/diseases/novelcoronavirus-2019.

Xia, Y., Wulan, N., Wang, K., \& Zhang, H. (2018). Detecting atrial fibrillation by deep convolutional neural networks. Computers in Biology and Medicine, 93, 84-92.

Yang, Y., Lu, Q., Liu, M., Wang, Y., Zhang, A., Jalali, N., \& Zhang, X. (2020). Epidemiological and clinical features of the 2019 novel coronavirus outbreak in China. medRxiv. 